\title{
REDES Y PROCESOS DE INNOVACIÓN TURISTICA EN LAS COMARCAS VITIVINÍCOLAS DE LA REGIÓN DE MURCIA: LAS RUTAS DEL VINO Y SUS CONSECUENCIAS EN LA TRANSFORMACIÓN DEL PAISAJE ${ }^{1}$.
}

\section{INNOVATION NETWORKS AND PROCESSES OF WINE TOURISM IN THE REGION OF MURCIA: WINE ROUTES AND ITS CONSEQUENCES IN THE CHANGING LANDSCAPE.}

\author{
Francisco José Morales Yago \\ Universidad Nacional de Educación a Distancia \\ fjmorales@geo.uned.es
}

Recibido: marzo, 2012.

Versión final aceptada: enero, 2013.

PALABRAS CLAVE: enoturismo, vino, desarrollo local, gastronomía, paisaje y patrimonio, denominación de origen.

KEY WORDS: wine tourism, wine, local development, food, landscape and heritage, designation of origin.

\section{RESUMEN}

Entre las modalidades más importantes del denominado turismo agrícola está el enoturismo o "turismo vitivinícola", su principal objetivo se centra en el descubrimiento de los espacios vitícolas y las distintas connotaciones paisajísticas y etnográficas que ello conlleva, así como la consolidación de un sistema productivo local que incluye una red de ciudades con igual orientación productiva.

El presente artículo tiene como objetivo estudiar las transformaciones territoriales derivadas de esta actividad productiva que no se limita al sector primario sino que ha incorporado elementos de terciarización del espacio agrícola que poco tienen que ver con el tradicional uso dedicado a la obtención de vino, de hecho han aparecido innovaciones reflejadas en la estética de las bodegas, creación de museos del vino, visitas a empresas, alojamientos, restaurantes o visitas a espacios culturales próximos. Como muestra tangible de esta realidad transformadora del paisaje y la economía local estudiaremos las actuales rutas del vino de la Comunidad de Murcia: Bullas, Jumilla y Yecla.

\footnotetext{
1 El presente artículo procede de la comunicación científica presentada en las Jornadas de Estudios Regionales: Redes y sus territorios: Incidencia en el desarrollo regional. AGE. Sevilla, Junio de 2012.
}

ISSN: 0212-8594 ISSN-e: 2340-2776 № DOI: http://dx.doi.org/10.12795/rea.2012.i29.04 

del vino y sus consecuencias en la transformación del paisaje.

\section{ABSTRACT}

Among the most important forms of so-called agricultural tourism is he wine or "wine tourism", its main focus is on the discovery of the area under vines and the different connotations and ethnographic landscape that entails, and the consolidation of local production system which includes a network of cities with the same productive orientation.

This article aims to study the territorial changes resulting from this productive activity is not limited to the primary sector but has incorporated elements of outsourcing of agricultural land that have little to do with the traditional use dedicated to obtaining wine, in fact innovations have appeared reflected in the a esthetics of the wineries, wine museum building, visits to companies, hotels, restaurants and visits to cultural venues nearby. As tangible sign of this reality, transforming the landscape and the local economy will study the current wine routes of the Community of Murcia: Bullas, Jumilla and Yecla. 


\section{EL ENOTURISMO, UN ELEMENTO INNOVADOR PARA LA DINAMIZACIÓN DE LOS ESPACIOS AGRARIOS.}

La Carta Europea del Enoturismo del año 2005, desarrollada por la Red Europea de Ciudades del Vino - Recevin- al amparo del proyecto europeo VINTUR define al enoturismo: "Como todas aquellas actividades y recursos turísticos y de ocio y tiempo libre relacionados con las culturas, materiales e inmateriales, del vino y gastronomía autóctona de sus territorios".

En dicha carta se indican tres elementos esenciales relacionados con la dinamización de los espacios relacionados con la agricultura vitivinícola: el primero sería la sostenibilidad ambiental del territorio, el segundo la promoción de una cooperación eficaz entre zonas vitivinícolas y por último la difusión de una auténtica cultura del vino que tenga una gestión integral, correcta y esmerada.

En el enoturismo también podríamos hablar de dos objetivos generales (Goyet, P., 2010) un cualitativo y otro cuantitativo. A través de la promoción del vino, tiene por objetivo aumentar el volumen de ventas directas al público y al mismo tiempo incrementar las visitas a las regiones vinícolas que por lo general suelen estar alejadas de los circuitos tradicionales de Sol y Playa, ofreciendo otras alternativas relacionadas con visitas culturales, descubrimiento del patrimonio artístico y natural sin olvidar un factor de gran peso: la gastronomía que acompañará a los vinos de la zona visitada.

Francia fue el primer país en potenciar este tipo de turismo desde hace más de 50 años, con la puesta en marcha de rutas del vino en regiones como Alsacia, Borgoña o Burdeos; las cuales ha sabido combinar un importante número de actividades que lógicamente giran alrededor de la cultura del vino, por ejemplo los museos enológicos, recorrido por bodegas, transporte aéreo sobre los viñedos a través de globos aerostáticos o avionetas o puesta en marcha de hoteles rurales, etc. (Parenteau, A., 2010)

Teniendo como referencia al país galo, otros territorios como España, Italia o Portugal están promoviendo esta actividad que se encuentra en plena fase de consolidación, al mismo tiempo que está ayudando a la promoción turística de espacios que no habían gozando de una actividad turística reconocida, aun gozando de posibilidades importantes como serían la belleza de los paisajes, la presencia de importantes recursos patrimoniales y de una rica y variada gastronomía (Zárate, A., 2010)

Entre los setenta y cuatro municipios europeos que componen la red europea de ciudades del vino, destacan de forma muy amplia dos países: Italia con veintidós y España que se encuentra a la cabeza con un total de veinticuatro, llama poderosamente la atención el caso de Francia, que siendo el país con mayor desarrollo

ISSN: 0212-8594 ISSN-e: 2340-2776 № DOI: http://dx.doi.org/10.12795/rea.2012.i29.04

REA 29 (2012):83-104

http://www.publius.us.es/estudios_andaluces 
del enoturismo solamente han queda asociado cinco municipios. La promoción y puesta en valor de estas rutas del vino ha dependido mayoritariamente del interés de los municipios que han visto en ello una importante oportunidad de negocio.

Gráfico № 1. Listado de municipios de la Red Europea de Ciudades del Vino.

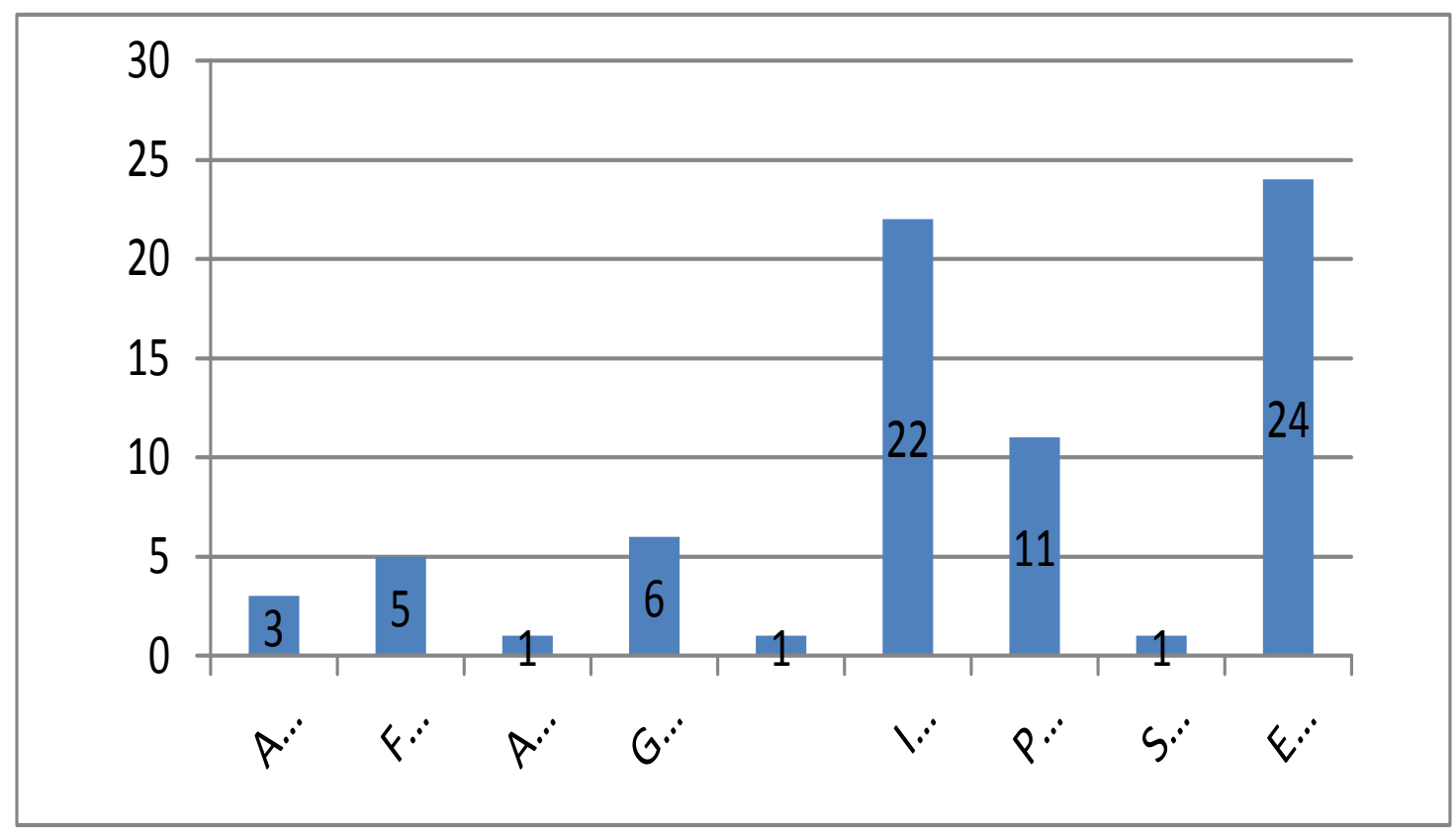

Fuente: Acevin 2012.

\section{ESPAÑA, UN PAÍS DE GRANDES POSIBILIDADES PARA EL ENOTURISMO.}

España reúne un total de 67 denominaciones de Origen y de las 17 Comunidades autónomas que configuran el estado español en quince (exceptuando Asturias y Cantabria) existe alguna de estas denominaciones, ello indica que la producción vinícola es un actividad importante en nuestro país, aunque evidentemente en no todas las denominaciones el paso al enoturismo ha tenido la misma intensidad, incluso en algunas de ellas los pasos dados han sido incipientes, mientras que otros espacios gozan de un reconocimiento y tradición que arranca desde hace muchas décadas.

No se entiende el enoturismo sin el vino, pero tampoco se entiende la búsqueda del patrimonio, la belleza de los paisajes o el maridaje de los vinos con el plato típico de la tierra, de ahí que podamos afirmar que la cultura del vino es integradora y se perfila como uno de los mejores atractivos del turismo de interior en nuestro país. 

del vino y sus consecuencias en la transformación del paisaje.

España es un país de enormes posibilidades turísticas, no podemos olvidar que el tejido industrial español en general ha caído en una falta de competitividad, se requiere ante esta realidad, la búsqueda de negocios alternativos que puedan ser lo suficientemente rentables para crear nuevos puestos de trabajo y una salida sostenible antes los retos que ha marcado la globalización y que tanto han perjudicado a nuestra economía en el sector productivo industrial. El turismo, y en el caso que nos ocupa: el enoturismo se perfila como una posibilidad de dotar a los espacios agrarios de una dinámica plurifuncional, diversificando el espacio y complementado la producción agrícola con otras actividades relacionadas con el ocio.

Figura no 1. Regiones vinícolas españolas productoras de vinos con Denominación de Origen.

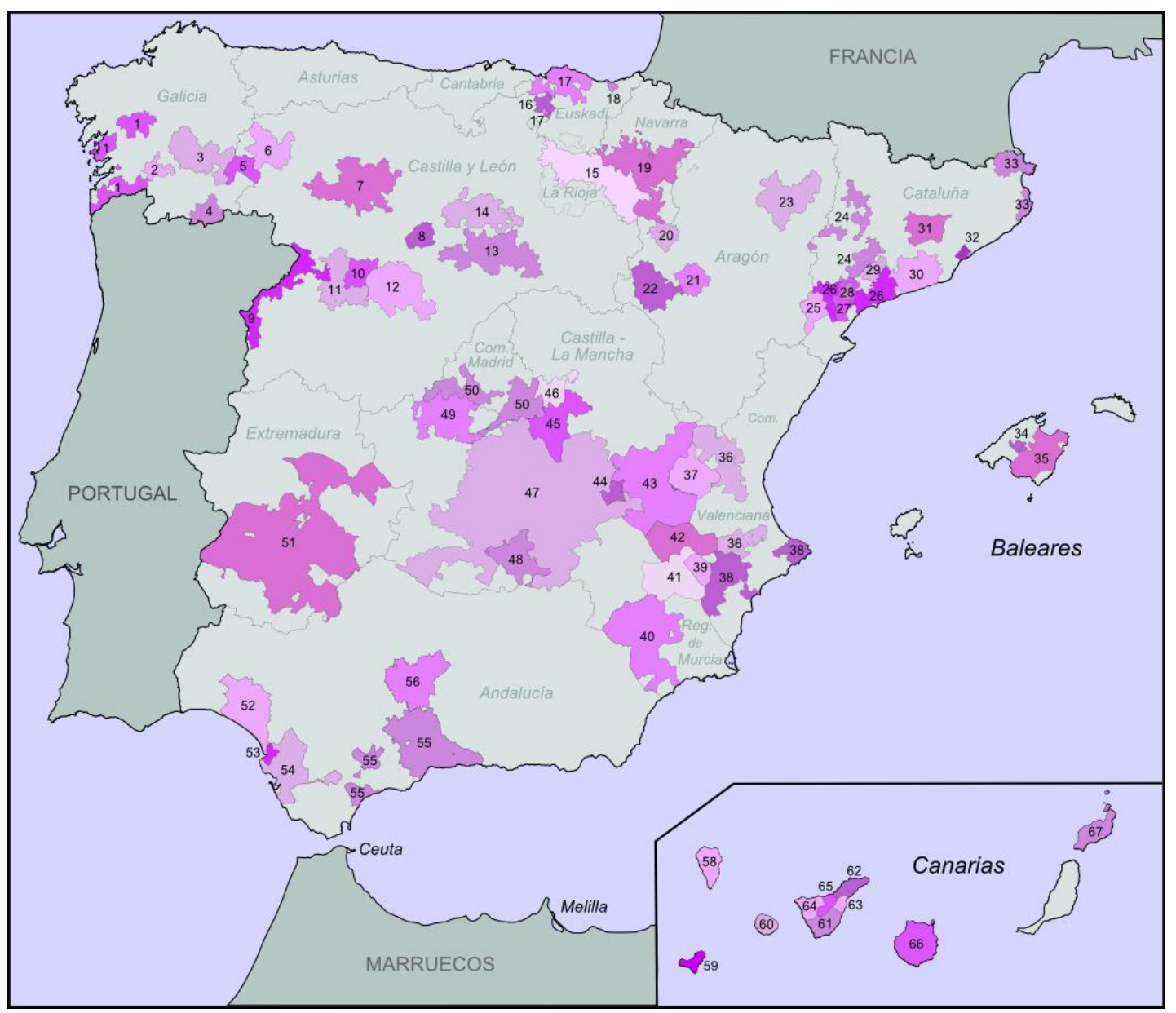

Fuente: http://upload.wikimedia.org/wikipedia/commons/5/52/Vinos_DO_de_Espa\%C3\%B1a.png

ISSN: 0212-8594 ISSN-e: 2340-2776 № DOI: http://dx.doi.org/10.12795/rea.2012.i29.04 REA 29 (2012):83-104

http://www.publius.us.es/estudios_andaluces 


\section{LA PROMOCIÓN DEL ENOTURISMO EN ESPAÑA: LA ASOCIACIÓN DE RUTAS DEL VINO.}

Acevin es la asociación española de ciudades del vino, su estrategia consiste en valorizar el potencial endógeno de las zonas y ciudades donde el cultivo y elaboración del vino han sido y son la base productiva en torno a las cuales se han configurado históricamente. Las ciudades del vino promueven la valorización de este sector con el fin de potenciarlos como motor de otras actividades (desarrollo turístico, promoción económica, empleo, etc.) La presencia del vino como base productiva agroindustrial actúa como eje articulador de actividades diversas: a las actividades tradicionales vitícolas y vinícolas de cultivo, elaboración y distribución del vino se agregan un conjunto de actividades conexas y complementarias que podrían agruparse en cuatro facetas:

a) El estimulo y desarrollo de las actividades turísticas, tales como el marketing turístico, la creación y mantenimiento de museos del vino, restauración, hoteles y alojamientos, tiendas especializadas, pequeño comercio y rutas del vino.

b) La promoción cultural del territorio a través de la rehabilitación, recuperación y promoción del patrimonio arquitectónico e histórico y fiestas populares.

c) Actividades comerciales, publicaciones, organización de ferias, actos e incorporación de las nuevas tecnologías para la comercialización y promoción

d) La protección de los recursos naturales, por ejemplo depuración y reciclaje de aguas o desarrollo sostenible del territorio.

La actividad más importante de Acevin es la creación y mantenimiento de las denominadas "Rutas del vino". En la actualidad en el catálogo de rutas del vino diseñado por Acevin tenemos un total de 17 rutas certificadas en once comunidades autónomas y otras 9 que lo harán en próximas fechas, ello supone la participación de casi mil empresas repartidas en 160 ciudades y más de 400 bodegas.

En relación a estas rutas certificadas encontramos nombres muy conocidos: Bullas, La Mancha, Campo de Borja, Penedés, Jumilla, Lleida, Jerez, Montilla-Moriles, Navarra, O Ribeiro, Rías Baixas, Ribera del Duero, Rioja Alavesa, Somontano, Tacoronte, UtielRequena y El Bierzo. 
Francisco José Morales Yago

Redes y procesos de innovación turística en las comarcas vitivinícolas de la región de Murcia: Las rutas del vino y sus consecuencias en la transformación del paisaje.

Gráfico no 2. Rutas del vino certificadas en España por CC.AA.

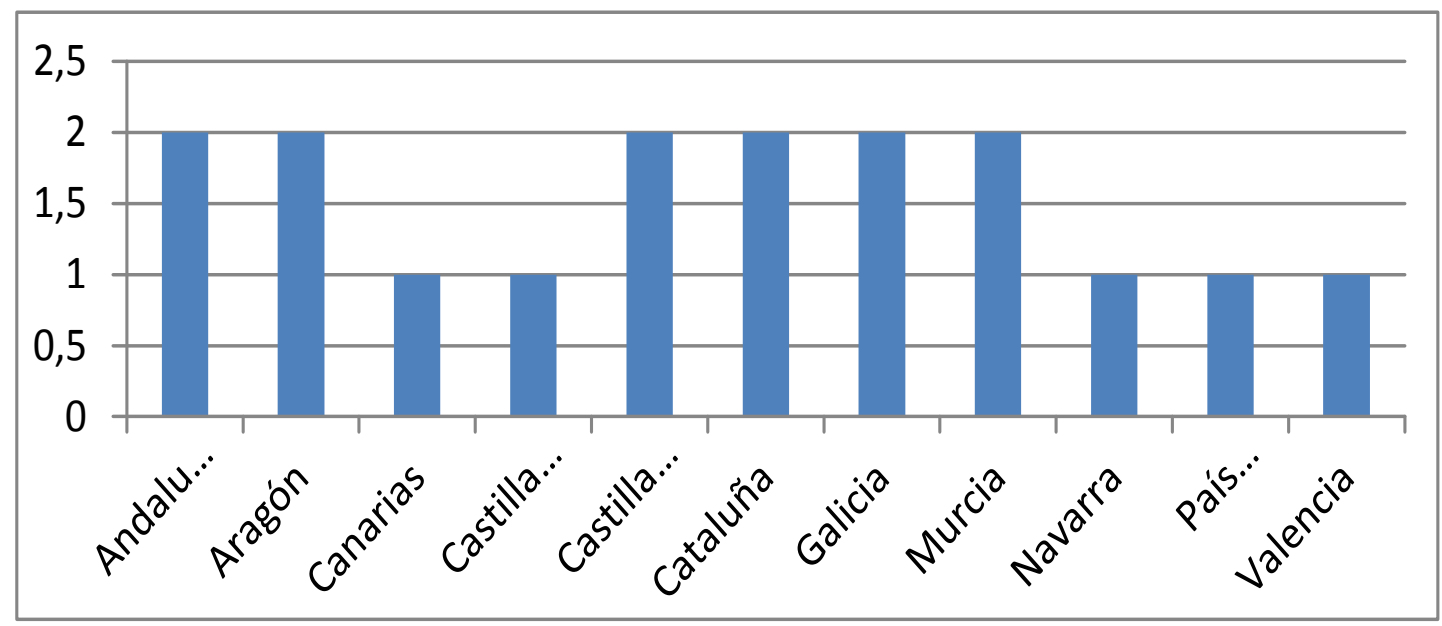

Fuente: Acevin 2012.

Cuadro nำ1. Otras rutas del Vino en proceso de certificación.

\begin{tabular}{|l|l|}
\hline Nombre de la ruta & Comunidad Autónoma \\
\hline Alicante & Valencia \\
\hline Condado de Huelva & Andalucía \\
\hline Ribera de Guadiana & Extremadura \\
\hline Rioja Alta & La Rioja \\
\hline Ycoden Daute Isora & Canarias \\
\hline Cariñena & Aragón \\
\hline Yecla & Murcia \\
\hline Txakolí & País Vasco \\
\hline Rueda & Castilla-León \\
\hline
\end{tabular}

Fuente: Acevin 2012.

ISSN: 0212-8594 ISSN-e: 2340-2776 № DOI: http://dx.doi.org/10.12795/rea.2012.i29.04 REA 29 (2012):83-104

http://www.publius.us.es/estudios_andaluces 
Figura no 2 Rutas del Vino en España

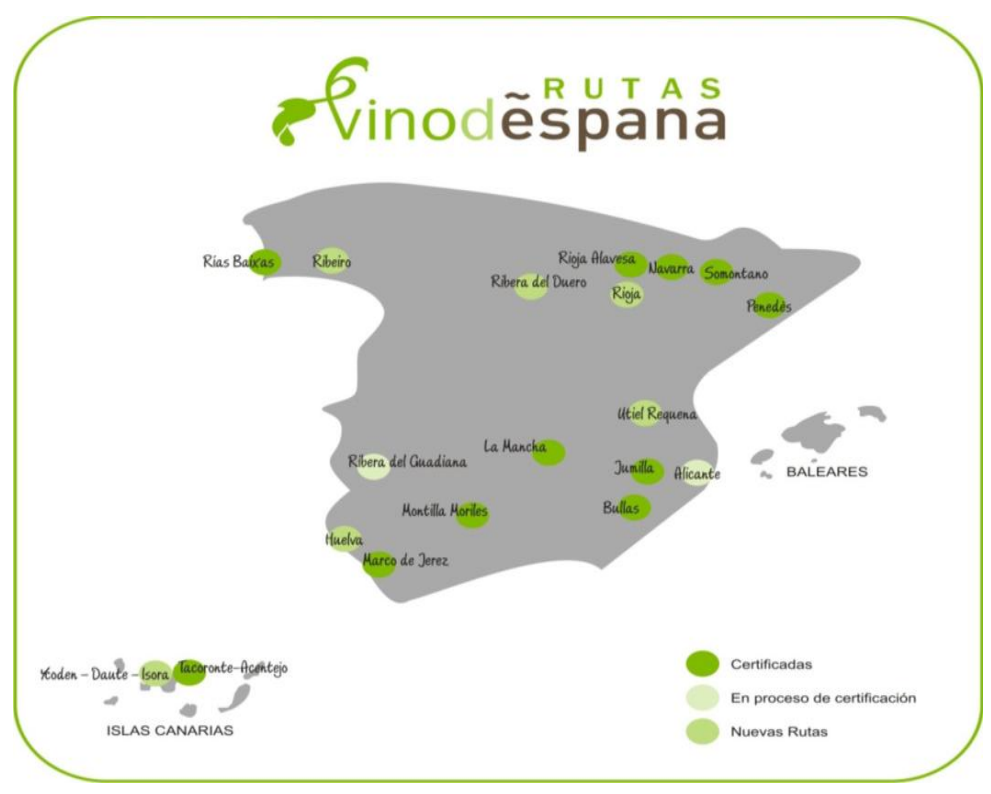

Fuente: Acevin 2011.

\section{CLAVES PARA EL DESARROLLO DEL ENOTURISMO EN LAS DENOMINACIONES DE ORIGEN DE LA REGIÓN DE MURCIA. ANÁLISIS DAFO.}

El cultivo más importante en las tres comarcas objeto de estudio es el viñedo, la producción de vino ha provocado una búsqueda en la exportación como una importante salida para dar sostenibilidad económica, de hecho en certámenes vinícolas internacionales no faltan los vinos de la Región de Murcia, esta circunstancia ha generado que la producción vinícola generalmente consumida en territorio nacional haya acaparado mercados internacionales. Tras las diversas reestructuraciones del viñedo para adaptarse a las demandas de la U.E. actualmente la superficie cultivada se encuentra estabilizada, no se vislumbra en el futuro posibles arranques; más bien la posibilidad de nuevas plantaciones que puedan conllevar perspectivas de expansión económica ante el actual crisis del modelo industrial existente que tiene graves dificultades para competir con los mercados emergentes de oriente tales como China o los "dragones asiáticos". La internacionalización de los vinos murcianos se encuentra en diversos países importadores pertenecientes a la U.E. como Alemania, Reino Unido, Bélgica, Suiza, Noruega, Francia, Holanda, Dinamarca, Austria, Suecia y Finlandia; aunque también están apareciendo nuevos mercados en América (Canadá y EE.UU.) y en Asia (Japón y China).

ISSN: 0212-8594 ISSN-e: 2340-2776 № DOI: http://dx.doi.org/10.12795/rea.2012.i29.04 REA 29 (2012):83-104

http://www.publius.us.es/estudios_andaluces 

del vino y sus consecuencias en la transformación del paisaje.

Cuadro no 3 Evolución superficie total de las explotaciones agrarias en Bullas, Jumilla y Yecla.

\begin{tabular}{|c|c|c|c|c|c|c|}
\hline & \multicolumn{2}{|c|}{$\begin{array}{l}\text { Término municipal de } \\
\text { Bullas }\end{array}$} & \multicolumn{2}{|c|}{$\begin{array}{l}\text { Término municipal de } \\
\text { Jumilla }\end{array}$} & \multicolumn{2}{|c|}{$\begin{array}{l}\text { Término municipal de } \\
\text { Yecla }\end{array}$} \\
\hline & Secano & Regadío & Secano & Regadío & Secano & Regadío \\
\hline $\begin{array}{c}2004 \\
\%\end{array}$ & $\begin{array}{l}585 \\
7,11\end{array}$ & $\begin{array}{l}4 \\
0,04\end{array}$ & $\begin{array}{l}17578 \\
18,14\end{array}$ & $\begin{array}{l}3336 \\
3,44\end{array}$ & $\begin{array}{l}8405 \\
13,87\end{array}$ & $\begin{array}{l}5605 \\
9,25\end{array}$ \\
\hline $\begin{array}{l}2005 \\
\%\end{array}$ & $\begin{array}{l}605 \\
7,36\end{array}$ & $\begin{array}{l}4 \\
0,04\end{array}$ & $\begin{array}{l}17461 \\
18,01\end{array}$ & $\begin{array}{l}3336 \\
3,44\end{array}$ & $\begin{array}{l}8405 \\
13,87\end{array}$ & $\begin{array}{l}5635 \\
9,30\end{array}$ \\
\hline $\begin{array}{l}2006 \\
\%\end{array}$ & $\begin{array}{l}605 \\
7,36\end{array}$ & $\begin{array}{l}4 \\
0,04\end{array}$ & $\begin{array}{l}17461 \\
18,01\end{array}$ & $\begin{array}{l}3336 \\
3,44\end{array}$ & $\begin{array}{l}8405 \\
13,87\end{array}$ & $\begin{array}{l}5635 \\
9,30\end{array}$ \\
\hline $\begin{array}{l}2007 \\
\%\end{array}$ & $\begin{array}{l}605 \\
7,36\end{array}$ & $\begin{array}{l}4 \\
0,04\end{array}$ & $\begin{array}{l}16810 \\
17,34\end{array}$ & $\begin{array}{l}3336 \\
3,44\end{array}$ & $\begin{array}{l}7764 \\
12,81\end{array}$ & $\begin{array}{l}5635 \\
9,30\end{array}$ \\
\hline $\begin{array}{l}2008 \\
\%\end{array}$ & $\begin{array}{l}605 \\
7,36\end{array}$ & $\begin{array}{l}4 \\
0,04\end{array}$ & $\begin{array}{l}16630 \\
17,16\end{array}$ & $\begin{array}{l}3181 \\
3,28\end{array}$ & $\begin{array}{l}7584 \\
12,52\end{array}$ & $\begin{array}{l}5431 \\
8,96\end{array}$ \\
\hline
\end{tabular}

Fuente: Anuario Estadístico de la Región de Murcia.

Para valorar de manera precisa la situación actual del enoturismo en la Región de Murcia y llegar de la forma más objetiva posible a un diagnóstico que nos permita superar los peligros que se ciernen sobre el mismo, hemos recurrido a la utilización del llamado análisis DAFO. Mediante esta metodología se ponen de relieve las debilidades que presentan en este sector de actividad económica y las amenazas que sufre.

La situación actual es difícil ya que en ambos casos, si no se toman con decisión medidas correctoras, los puntos débiles pueden contrarrestar los puntos fuertes de este incipiente sector económico y las oportunidades que se le presentan. La metodología empleada ha consistido en la aplicación de esta herramienta de trabajo a doce empresas ubicada en las tres denominaciones de origen, para ello se recurrió al envío de un cuestionario que en la mitad los casos se hizo directamente a través una entrevista personal con gerentes de bodegas, autoridades públicas y encargados de oficinas de turismo, los datos obtenidos a través de las encuestas y su posterior tabulación nos han permitido disponer de una visión aproximada de la actual situación del sector. Salvo excepciones la participación de los encuestados ha sido plena. A continuación señalamos los resultados obtenidos, que esperamos puedan servir de referencia como un análisis actual y de proyección futura.

ISSN: 0212-8594 ISSN-e: 2340-2776 № DOI: http://dx.doi.org/10.12795/rea.2012.i29.04

REA 29 (2012):83-104

http://www.publius.us.es/estudios_andaluces 


\section{Cuadro no 4. Debilidades del sector del vino en la Región de Murcia.}

1. La imagen de los vinos de Murcia es poco conocida a nivel internacional, aunque la venta de vinos ha llegado a países como EE.UU., Japón o Australia continua siendo el mercado nacional el principal cliente, ello repercute directamente en que existan pocas visitas a las rutas de vino murcianas por parte de extranjeros.

2. Todavía más de la mitad de bodegas no tiene previstas las visitas ni han acondicionado sus instalaciones para esta actividad complementaria.

3. Existe poca difusión a nivel de prensa y marketing de las rutas del vino de Murcia, de hecho faltan esfuerzos para una adecuada señalización tanto en las ciudades como en las posibles zonas en el medio rural.

4. El escaso turismo que visita estas zonas no tiene como prioridad la cultura del vino ya que existen otros atractivos de más peso, esencialmente en los municipios de Jumilla y Yecla. En el caso de Bullas el atractivo del producto turístico relacionado con el vino es más potente y prioritario por parte de los visitantes.

5. La actual crisis económica está perjudicando la llegada de fondos públicos y privados que incidan en la promoción turística de este sector.

6. A pesar de los esfuerzos realizados se detecta todavía una carencia de profesionales y guías turísticos especializados en la cultura del vino.

7. La capacidad hotelera de las tres rutas del vino es baja y tampoco se prevé en el futuro la construcción de nuevos alojamientos tanto urbanos como rurales, ello repercute en un turismo de visita diaria, en donde las pernoctaciones son muy escasas, lo que sin lugar a dudas perjudica al sector hostelero y de restauración.

\section{Cuadro no 5. Amenazas del sector del vino en la Región de Murcia.}

1. La visita a bodegas tiene un carácter limitado, ya que el acceso a las mismas queda en muchos casos restringido a la llegada de profesionales y clientes, no al público en general.

2. La imposibilidad en estos momentos de crisis de articular un proyecto turístico de la cultura del vino de gran eficiencia y de importantes resultados económicos y laborales.

3. El enoturismo se está convirtiendo en un sector que se extiende por todo el territorio nacional, puede darse el caso que las zonas más conocidas y con mayor nombre eclipsen otras rutas menos conocidas o poco desarrolladas..

4. La actual crisis económica influye en falta de subvenciones y ayudas públicas para el diseño y puesta en valor de las rutas del vino.

5. El enoturismo puede verse perjudicado por otras modalidades turísticas existentes en la zona, esencialmente por el turismo de sol y playa que nunca rebasa los 60 minutos de desplazamiento, cuestión que puede influir en la decisión final de los visitantes.

ISSN: 0212-8594 ISSN-e: 2340-2776 № DOI: http://dx.doi.org/10.12795/rea.2012.i29.04

REA 29 (2012):83-104

http://www.publius.us.es/estudios_andaluces 

del vino y sus consecuencias en la transformación del paisaje.

Cuadro no 6. Fortalezas del sector del vino en la Región de Murcia.

1. El paisaje agrario de las comarcas de Yecla, Jumilla y Bullas se mantiene conservado y ofrece un buen atractivo visual a lo largo de las diversas estaciones del año

2. En las tres rutas del vino se organizan a lo largo del año una serie de eventos y fiestas de gran arraigo relacionadas con la cultura del vino, en el caso de Bullas en septiembre se organiza la feria del vino, igual ocurre en Jumilla en el mes de Agosto, para el caso de Yecla es en el mes de mayo cuando se ensalza el vino a través de las fiestas de Primavera y San Isidro.

3. En los últimos años se ha hecho un gran esfuerzo en la recuperación de espacios etnográficos como sería el museo del vino en Bullas o la recuperación de bodegas en Jumilla y Yecla.

4. En las nuevas generaciones de bodegueros aparece una mentalidad empresarial en donde se tiene en cuenta la dimensión comercial de visitas turísticas y marketing de empresas.

5. Existe una tradición vinícola arraigada, el sector del vino forma parte de la cultura de estas comarcas y ha tenido continuidad en diversas épocas históricas.

Cuadro no 7. Oportunidades del sector del vino en la Región de Murcia.

1. La voluntad decidida de los ayuntamientos e instituciones públicas por impulsar el turismo del vino como el producto estrella de estas comarcas.

2. La aparición de nuevos operadores especializados en turismo enológico, aunque de escasa dimensión.

3. El desarrollo de planes de Dinamización Turística/ Producto específicos sobre el turismo del vino.

4. La integración de las tres denominaciones en el proyecto Rutas del Vino de España

5.Compatibilidad del turismo del vino con otros productos emergentes en Murcia y con la actividad de turismo de interior

El análisis DAFO refleja claramente que el sector del enoturismo en estas comarcas puede constituirse en un importante elemento de dinamización económica y constituye un sistema productivo local de gran proyección económica, es necesaria por tanto una adecuada promoción de esta actividad que se adapta al paisaje agrario y la tradición agrícola, para ello la unión de iniciativas comunes, formación de los agentes implicados y la colaboración entre empresas privadas, ayuntamientos y Consejería de Agricultura pueden ser elementos muy favorecedores de esta actividad económica.

\section{TRANSFORMACIONES EXPERIMENTADAS EN EL PAISAJE EN LAS DENOMINACIONES DE ORIGEN DE LA REGIÓN DE MURCIA DEBIDAS A LA ACTIVIDAD DEL ENOTURISMO.}

El enoturismo no deja de ser una actividad económica, lo que conlleva un uso del suelo y por tanto una transformación del espacio agrícola y urbano de los lugares en los que se desarrolla. En el caso de la Región de Murcia, observamos importantes transformaciones que vendrían a sintetizarse en diversos aspectos:

ISSN: 0212-8594 ISSN-e: 2340-2776 № DOI: http://dx.doi.org/10.12795/rea.2012.i29.04

REA 29 (2012):83-104

http://www.publius.us.es/estudios_andaluces 

del vino y sus consecuencias en la transformación del paisaje.

\subsection{INSTALACIONES VITIVINÍCOLAS.}

La remodelación de bodegas o construcción de nueva planta supone un gran esfuerzo de adaptación de un uso exclusivamente a espacio de producción a espacio de ocio y recreación, por ejemplo en las bodegas se integra las dependencias dedicadas a molturación, fabricación, depósito y embalaje de vino con otros espacios como restaurante, tienda de objetos y venta de vinos.

Fotografía no 1 Bodegas Elena (Jumilla)

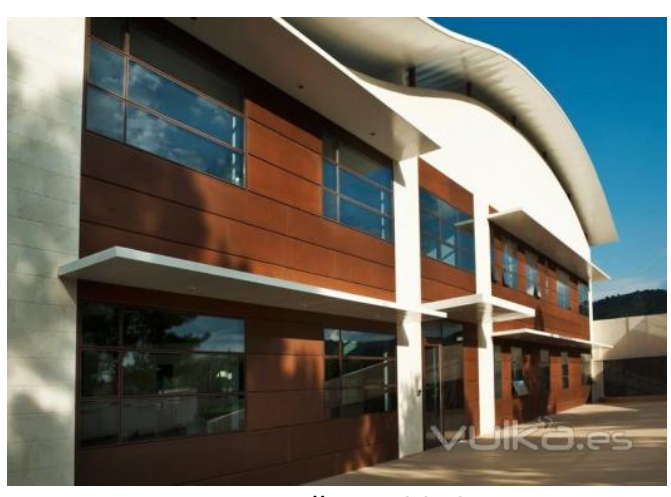

Fuente: www.vulka.es 2012
Fotografía no 2 Bodegas Señorío de Barahona (Yecla)

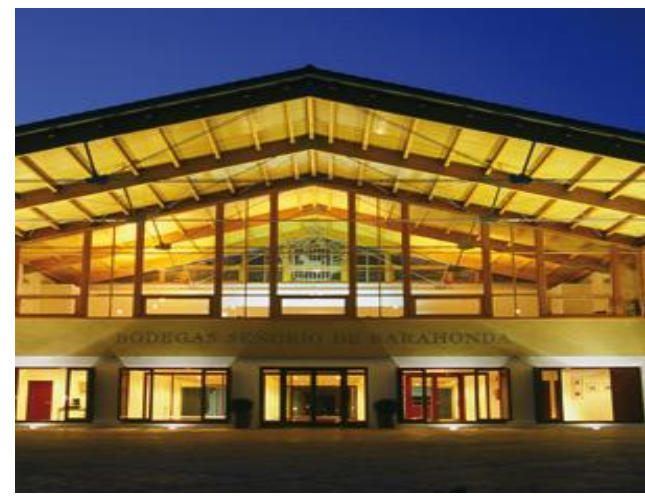

Fuente: www.barahonda.com. 2012

\subsection{SEÑALIZACIÓN DE LAS RUTAS.}

La cartelería es siempre un buen reclamo para orientar y atraer a los clientes, aparece tanto en los lugares próximos a instalaciones vitivinícolas en medio del campo como en las propias ciudades de referencia, de hecho incluso los posibles restaurantes o bares de copas se pueden identificar con un distintivo que asegure su vinculación con el vino.

\section{3. CREACIÓN DE MUSEOS.}

En este caso tenemos que destacar la creación del museo vitivinícola de Bullas, inaugurado en el año 2003 y reconocido en 2008 por la Consejería de Cultura, Juventud y Deportes en el Registro de Museos y colecciones museográficas de la Región de Murcia. Se trata de una importante contribución a la pervivencia del recuerdo de unas formas de vida en trance de desaparecer y al conocimiento de un mundo tan apasionante como el de la enología. Constituye un nuevo y sugerente atractivo turístico para la villa. Ubicado en una de las pocas bodegas tradicionales que han subsistido en perfecto estado de conservación, con sus bóvedas de ladrillo y tinajas semienterradas en el suelo, a lo largo de sus salas el visitante descubre la relación histórica que desde época romana esta tierra ha mantenido con la viticultura, así como el proceso técnico de elaboración del vino, en sus formas tradicionales y

ISSN: 0212-8594 ISSN-e: 2340-2776 № DOI: http://dx.doi.org/10.12795/rea.2012.i29.04

REA 29 (2012):83-104

http://www.publius.us.es/estudios_andaluces 
modernas, desde el momento de la vendimia hasta su almacenaje y envejecimiento en las bodegas, para culminar con didácticas explicaciones sobre las variedades de uvas y vinos y sobre las técnicas de la cata.

Figura no 3 Logos de las distintas rutas del vino y fiestas en Murcia.
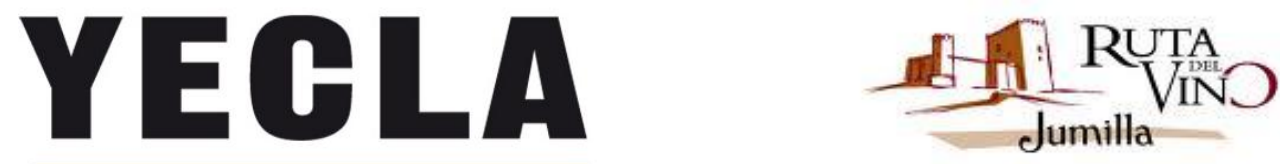

R U T A D E L vino

La bodega que alberga el Museo del Vino data del siglo XIX. Pertenecía inicialmente a la familia Melgares de Aguilar y, gracias a la conservación de gran parte de sus elementos originales, el edificio es el lugar ideal para ilustrar toda la actividad vinícola, actual y pasada, de la comarca que abarca la Denominación de Origen Bullas.

Sus más de $1.400 \mathrm{~m}^{2}$ ofrecen al visitante amplias posibilidades para recrearse en el ambiente cultural surgido en torno al vino. La producción anual de la bodega era de unos 350.000 litros de vino, repartidos en 112 tinajas, siendo a mediados del siglo XIX una de las de mayor tamaño y capacidad del viejo Reino de Murcia.

En el año 2006 se abrió un nuevo espacio relacionado con el vino, se trata de la casa “Don Pepe Marsilla. Bullas 1900", en donde se pretende rescatar una importante parte de la cultura local de la ciudad, recreando los modos de vida de los comienzo del siglo $X X$, al mismo tiempo que conservar el patrimonio históricos de la localidad representado en este edificio. El conjunto se compone de una zona residencial en donde vivían los antiguos señores de la casa, a lo que se añade la zona de labor en donde se elaboraba el vino y se almacenaba, de hecho quedan un total de 52 tinajas que tiene una capacidad para más de 140.000 litros de vino.

Por su parte en la localidad de Jumilla, se anuncia la intención de crear un gran museo del vino en una superficie de $900 \mathrm{~m} 2$, que formaba parte de una antigua bodega y ahora se encuentra en estado de abandono, esta iniciativa museística representa una clara intencionalidad de fomentar la cultura del vino, de hecho en la ciudad existe un museo etnográfico y en varias bodegas privadas se muestran objetos y utensilios relacionados con la actividad vinícola. 

del vino y sus consecuencias en la transformación del paisaje.

Fotografía no 3. Entrada al Museo de Bull.

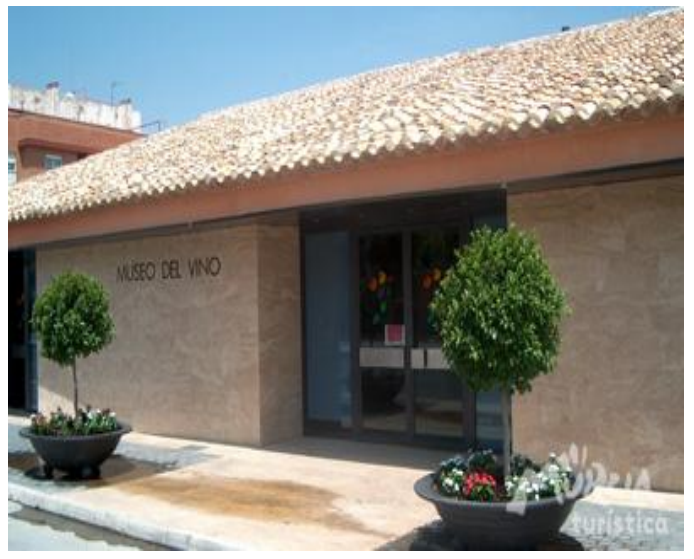

Fuente: http://www.museodelvino.bullas.es
Fotografía no 4. Interior del museo de Bullas.

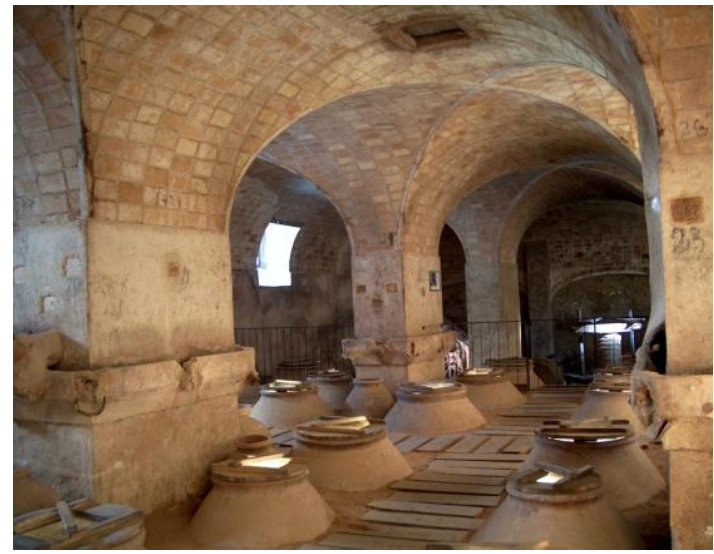

Fuente: http://www.museodelvino.bullas.es

\subsection{SOSTENIMIENTO DE LAS TIERRAS DE CULTIVO Y LA DIMENSIÓN ECOLÓGICA.}

El cultivo más importante en los tres espacios objeto de análisis es el viñedo, la producción de vino ha desarrollado la exportación de los caldos a diversas partes del mundo, de hecho en certámenes vinícolas no faltan los vinos de murcianos, esta circunstancia hace también que la producción vinícola generalmente consumida en territorio nacional haya acaparado mercados internacionales. El desarrollo rural trata de impulsar las actividades complementarias, como el turismo rural y la industria artesanal y asigna al campo la tarea de salvaguardar los recursos y el patrimonio natural, conservando y protegiendo el suelo fértil, los paisajes, los bosques y las riquezas ecológicas. De esta manera el mundo rural deja de ser un espacio exclusivamente agrario para convertirse en un espacio más diversificado, la globalización económica e incluso cultural contemporánea aparecen en el espacio agrario.

El cultivo del viñedo es poco consumidor de agua, lo cual refuerza la idea de adaptar las circunstancias climáticas y disponibilidades hídricas, siempre escasas, a unos cultivos sostenibles y poco agresivos con el medio ambiente; afortunadamente la viticultura en estas tierras reúne esas peculiaridades y salvo la capacidad de comercialización y competitividad de estos productos en todo lo referente a condiciones medioambientales, el cultivo de la vid es un magnífico ejemplo en el pasado y futuro de estas tierras, un cultivo que se introdujo en época romana y permanece dos mil años después, demuestra su perfecta adaptabilidad al suelo y la actividad humana que ahora más que nunca aglutina la actividad tradicional productiva con una nueva dinámica terciaria, lo cual pone de relieve que las diferencias entre los modos de vida del campo y la ciudad están siendo borrados.

ISSN: 0212-8594 ISSN-e: 2340-2776 № DOI: http://dx.doi.org/10.12795/rea.2012.i29.04

REA 29 (2012):83-104

http://www.publius.us.es/estudios_andaluces 


\subsection{VINCULACIÓN DE LA RUTA DEL VINO A LA VISITA DEL PATRIMONIO LOCAL.}

La visita a cada ruta del vino significa conocer y disfrutar el patrimonio local de la zona, de hecho se asocia a las diferentes rutas los principales museos, actos culturales o monumentos de interés histórico artístico que para el caso de Jumilla y Yecla son notables, y para Bullas se identifica con importantes ciudades que están próximas y guardan un gran patrimonio cultural como serían la ciudad santa de Caravaca de la Cruz o el casco histórico de la villa de Cehegín.

Fotografía n5 Imagen de Jumilla.

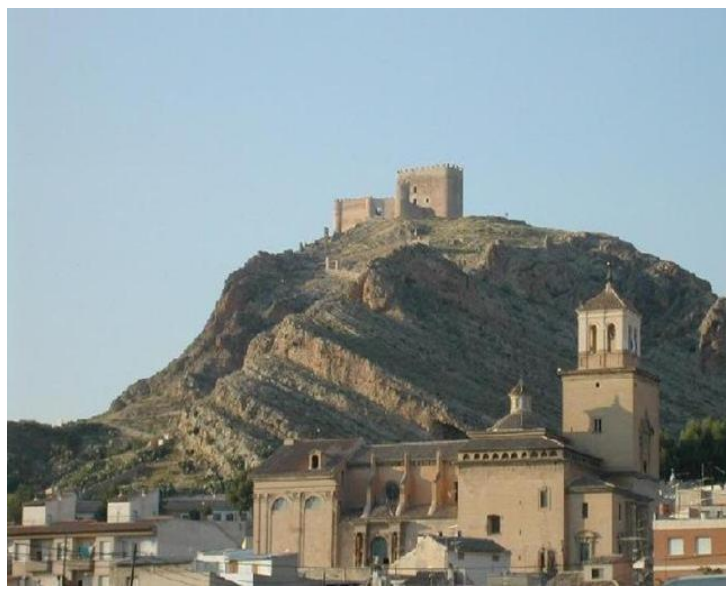

Fuente: Ruta del vino de Jumilla (2012)
Fotografía n 6 Basílica "La Purísima” de Yecla.

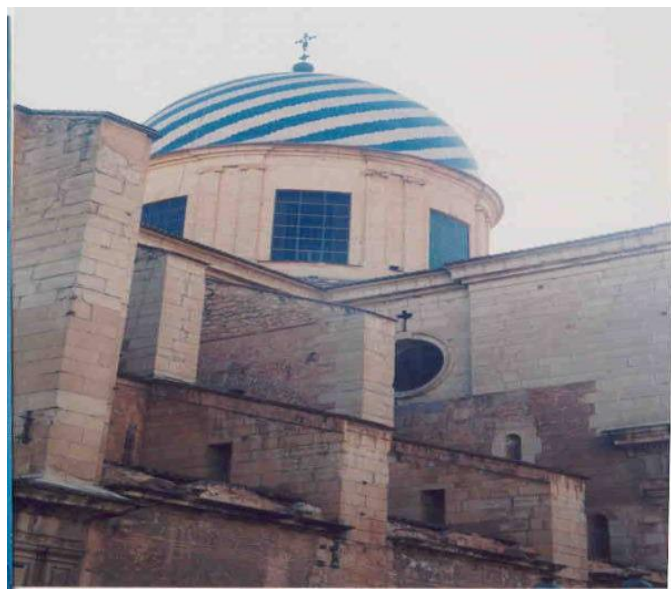

Fuente: F. Morales (2012)

\subsection{RECURSOS HOTELEROS.}

El sector hostelero de las denominaciones de origen estudiadas presentan grandes contrastes entre los lugares dedicados a las restauración y bares de vinos con la capacidad de alojamientos para pernoctar; abundan los primeros frente a una clara insuficiencia de los segundos, ello provoca la creación de un turismo de día, con un nivel de estancias muy corto, que no supera la media de 6 horas (Plan estratégico del turismo de Murcia). Los establecimientos hoteleros existentes tampoco son de gran nivel puesto que no existe ningún hotel de cuatro o cinco estrellas, sólo en Bullas y Jumilla encontramos en cada una de estas localidades un solo establecimiento de tres estrellas.

ISSN: 0212-8594 ISSN-e: 2340-2776 № DOI: http://dx.doi.org/10.12795/rea.2012.i29.04

REA 29 (2012):83-104

http://www.publius.us.es/estudios_andaluces 
Francisco José Morales Yago

Redes y procesos de innovación turística en las comarcas vitivinícolas de la región de Murcia: Las rutas del vino y sus consecuencias en la transformación del paisaje.

Cuadro no 2 Recursos hoteleros de las D.O.de Murcia (Año 2012).

\begin{tabular}{|l|c|c|c|}
\hline & D.O. Bullas & D.O. Jumilla & D.O. Yecla \\
\hline № alojamientos hoteleros & 2 & 4 & 2 \\
\hline Alojamientos rurales & 22 & 4 & 3 \\
\hline № Plazas alojamientos total & 164 & 141 & 158 \\
\hline № de Restaurantes & 5 & 13 & 8 \\
\hline Bodegas & 11 & 46 & 14 \\
\hline Bares de vinos & 9 & 11 & 0 \\
\hline Camping no plazas & 350 & 0 & \\
\hline
\end{tabular}

Fuente: Plan Dinamización turística de Murcia (2007-2013).

De las bodegas existentes en las denominaciones en las distintas denominaciones de origen es importante destacar su localización por municipios:

Cuadro no 3 Distribución de las bodegas por municipios y denominaciones de Origen.

\begin{tabular}{|l|l|l|l|}
\hline \multicolumn{1}{|c|}{ Municipios } & D.O. Bullas & D.O. Jumilla & D.O. Yecla \\
\hline Bullas & 7 & & \\
\hline Cehegín & 2 & & \\
\hline Moratalla & 1 & & \\
\hline Lorca & 1 & & \\
\hline Jumilla & & 33 & \\
\hline Tobarra & & 2 & \\
\hline Albatana & & 1 & \\
\hline Fuentealamo & & 2 & \\
\hline Ontur & & 2 & \\
\hline Montealegre del Castillo & & 2 & \\
\hline Pinoso & & & \\
\hline Yecla & & & \\
\hline Fuente: & & & \\
\hline
\end{tabular}

Fuente: Consejos Reguladores de Denominación de Origen y elaboración Propia.

ISSN: 0212-8594 ISSN-e: 2340-2776 № DOI: http://dx.doi.org/10.12795/rea.2012.i29.04

REA 29 (2012):83-104

http://www.publius.us.es/estudios_andaluces 

del vino y sus consecuencias en la transformación del paisaje.

\subsection{PATRIMONIALIZACIÓN DE LAS FIESTAS DEDICADAS AL VINO.}

En las tres localidades objeto de estudios, se vincula de una forma muy significativa la celebración de fiestas con la exaltación del vino y las tareas agrarias, en el municipio de Bullas, en el mes de septiembre se realizan las denominadas: "Fiestas del Vino" en donde se recuperan tareas antiguas como la "pisada" de uvas o concurso de catas, ello genera visitas y un importante dinamismo económico para la ciudad.

Fotografía nำ Cartel fiestas de Bullas.

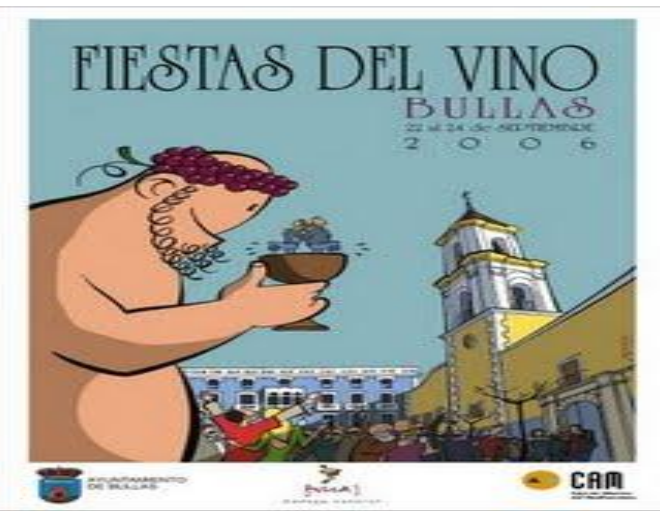

Fuente: web ayuntamiento de Bullas
Fotografía no 8 "Pisada tradicional de uva".

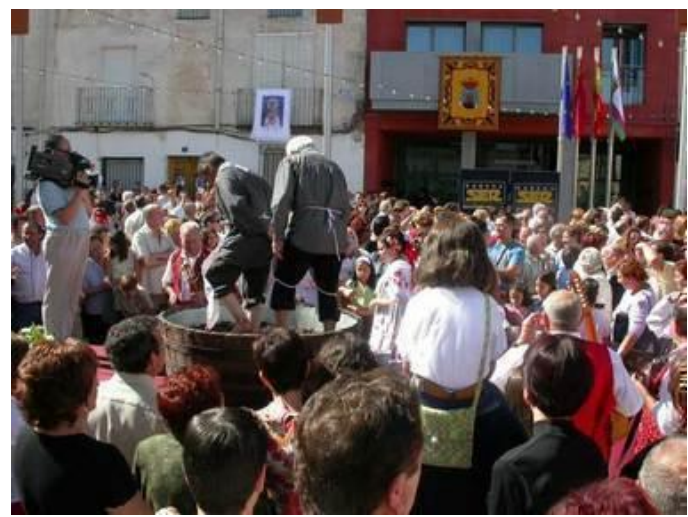

Fuente: web ayuntamiento de Bullas

En el caso de Jumilla, en el mes de agosto coincide la feria, celebración de Moros y Cristianos y fiestas del vino, no cabe dudas que el conjunto de estos eventos genera para la localidad la visita de turistas y una proyección importante de la ciudad a nivel regional; la "cabalgata del vino" o la "fuente del vino" son espectáculos de gran atractivo turístico que atraen a miles de personas. También en una pedanía próxima Jumilla: "La Fuente del Pino", en el mes de octubre se recrean las denominadas: "Estampas rurales", en donde se presentan las tradicionales actividades del campo antes de la llegada del tractor y demás herramientas posteriores.

Finalmente en el municipio de Yecla, las fiestas dedicadas al vino se celebran en el mes de mayo coincidiendo con las denominadas "Fiestas de Primavera y San Isidro", destaca la cabalgata de carrozas engalanadas con motivos rurales y el concurso de catas de vino siempre asociado a la gastronomía local, la ciudad en esto días duplica la población, lo que genera un importante movimiento económico en el sector hostelero, al mismo tiempo los visitantes tienen la oportunidad de conocer el patrimonio histórico-artístico de la ciudad, destacando las visitas a museos y edificios singulares.

ISSN: 0212-8594 ISSN-e: 2340-2776 № DOI: http://dx.doi.org/10.12795/rea.2012.i29.04

REA 29 (2012):83-104

http://www.publius.us.es/estudios_andaluces 
Redes y procesos de innovación turística en las comarcas vitivinícolas de la región de Murcia: Las rutas del vino y sus consecuencias en la transformación del paisaje.

Fotografía no 9. Fuente del vino. Jumilla. Fotografía № 10. Cabalgata del vino Jumilla.

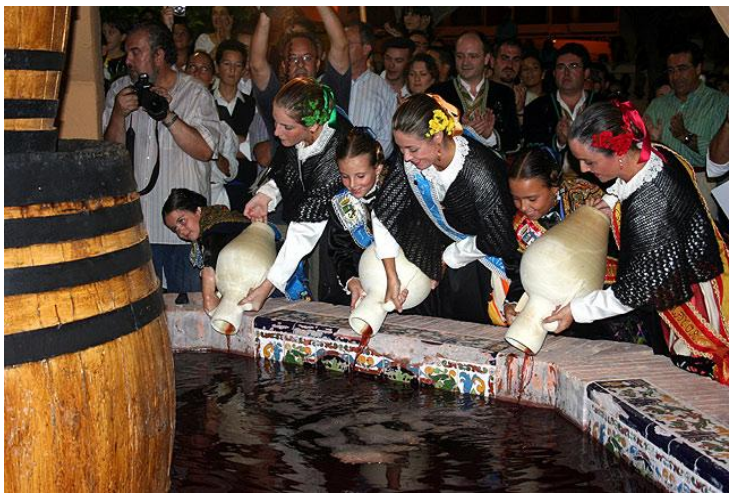

Fuente: web ayuntamiento de Jumilla

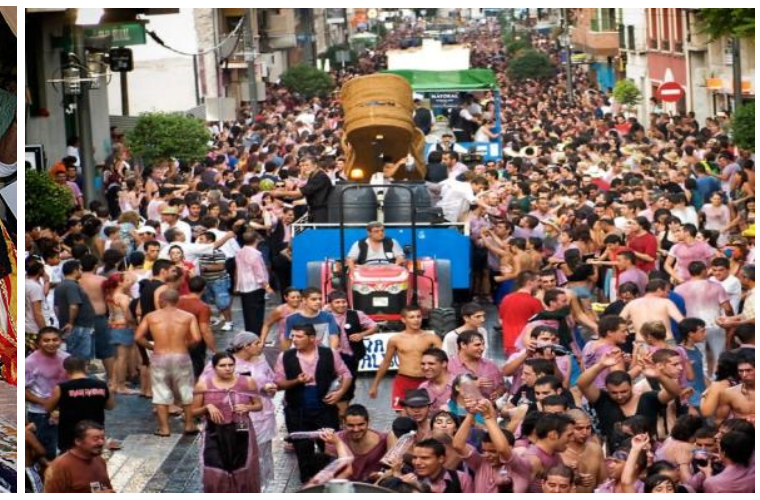

Fuente: web ayuntamiento de Jumilla
Fotografía no11 Carrozas de papel. Yecla

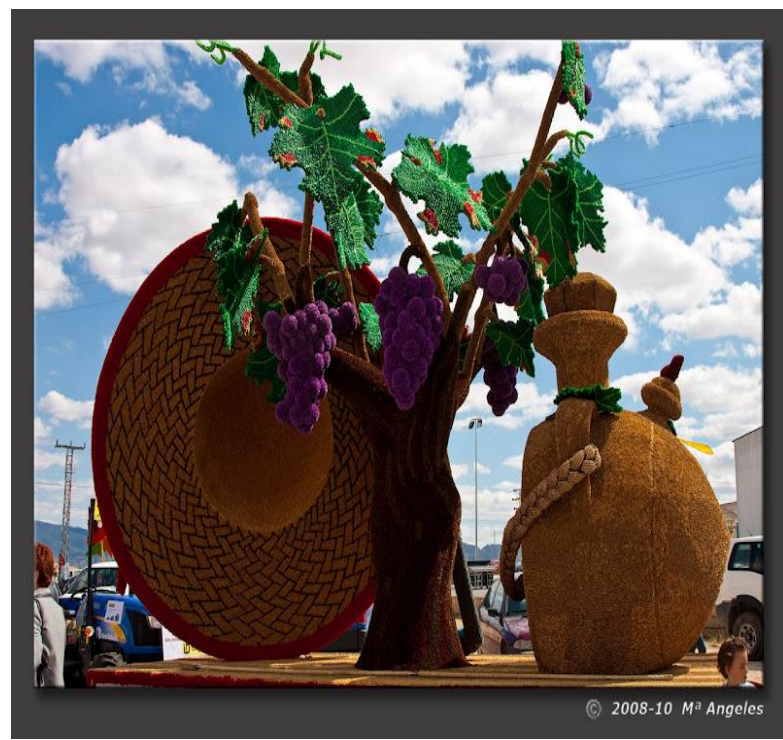

Fuente: web ayuntamiento de Yecla (2011)
Fotografía no 12. Fiestas de San Isidro. Yecla

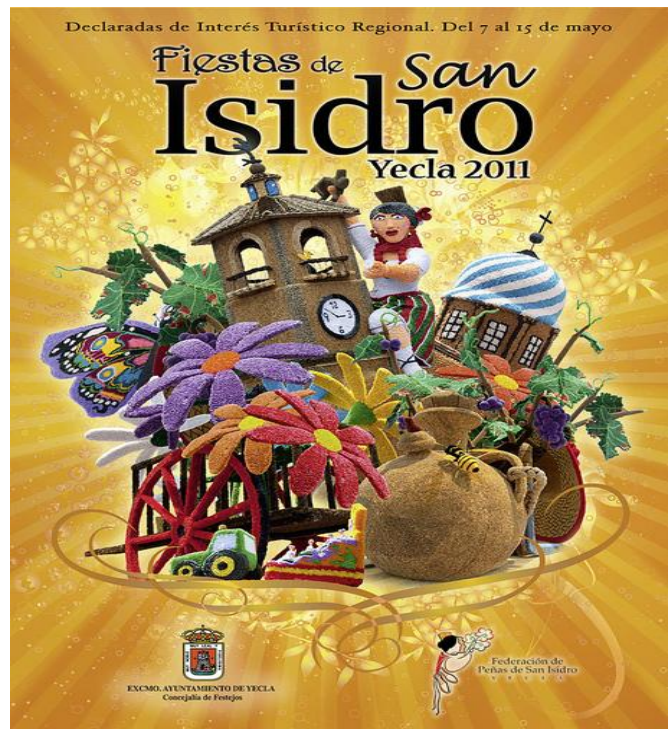

Fuente: web ayuntamiento de Yecla (2011)

\section{CONCLUSIONES.}

Las conclusiones de este trabajo pretenden recoger los aspectos más relevantes de este trabajo y se reseñan a continuación:

1.El sector agrario siempre ha ocupado un papel muy importante en la economía local de las ciudades objeto de estudio, esencialmente en los municipios de Bullas y Jumilla

ISSN: 0212-8594 ISSN-e: 2340-2776 № DOI: http://dx.doi.org/10.12795/rea.2012.i29.04

REA 29 (2012):83-104

http://www.publius.us.es/estudios_andaluces 

del vino y sus consecuencias en la transformación del paisaje.

ha sido el principal motor de la economía local, en el caso de Yecla su rápida industrialización a partir de los años 60 en la industria del mueble relegó a la agricultura a un plano secundario. Las rutas del vino suponen un más que evidente incremento en la generación de rentas en un momento de crisis aguda en las economías industriales que están siendo incapaces de hacer frente a la expansión de los productos industriales procedentes de Oriente, generalmente de peor calidad pero mucho más económicos en cuanto a precios.

2. La potencialidad del turismo rural, liderada por el enoturismo (Monnier, J.M. 2010) se perfila como un sector estratégico ya que está influyendo en aspectos como la visita a bodegas (Zárate, M. 2010) y posterior venta de productos, mayores ingresos en el sector hostelero y visita a lugares de interés cultural (Duboscq, M. 2010) que no han terminado de potenciarse en épocas anteriores.

3. Las tareas agrícolas y buenas prácticas en la tierra ofrecen la posibilidad de incrementar un mayor desarrollo sostenible y mejora en la protección del medio ambiente así como la preservación de especies de flora y fauna autóctonas.

4. Tecnificación, formación del personal e inversiones son elementos clave para afrontar la competencia de otros mercados emergentes que suponen un grave riesgo para la continuidad del sector agrario en estos municipios.

5. La expansión y puesta en valor del enoturismo supondrá la creación de nuevas fuentes de empleo y la posibilidad de complementar rentas para algunos agricultores, el caso del alquiler de casas rurales está todavía por desarrollar con fuerza en estas comarcas.

6. Las administraciones públicas a pesar de la actual situación económica de numerosos recortes presupuestarios deben racionalizar e incrementar las inversiones que potencien directamente cuestiones tan diversas como: mejora de infraestructuras y redes viarias, difusión y marketing de productos, negociación de acuerdos en la U.E., dinamización de planes de turismo interior o la negociación de acuerdos en la U.E., como por ejemplo el perjudicial tratado de la U.E. con Marruecos en febrero de 2012 que supone importantes problemas para los sectores hortofrutícolas de Andalucía, Murcia o Valencia por la competencia desleal en cuanto a los costes de mano de obra de los trabajadores o la falta de controles fitosanitarios en el país magrebí. Es imprescindible continuar e incrementar la colaboración entre empresas y ayuntamientos en el marco de las rutas del vino.

7. El análisis DAFO realizado destaca como posibles áreas de mejora para dinamizar el sector la internacionalización de los vinos de estas zonas, así como la continuidad en el esfuerzo de ofrecer un producto de calidad certificado por los grandes sumillers.

ISSN: 0212-8594 ISSN-e: 2340-2776 № DOI: http://dx.doi.org/10.12795/rea.2012.i29.04 REA 29 (2012):83-104 
8. Es preciso una mayor cohesión entre las tres denominaciones de origen para crear una única ruta del vino murciana, hasta el momento los puntos de encuentro y acercamiento entre las zonas son poco relevantes, existen más recelos que colaboración y búsqueda de metas conjuntas.

9. El enoturismo es una gran oportunidad para diversificar la economía en estos espacios, de hecho la actividad comercial, hostelera y de restauración en general se puede ver beneficiada.

10. Por último y de manera muy destacada entre las conclusiones, se estima oportuno la potenciación y búsqueda de nuevos productos turísticos relacionados alrededor de la cultura del vino, los paisajes culturales, la gastronomía; para ello se requiere un esfuerzo de creatividad e inversiones económicas tanto por parte de capital público como privado.

\section{BIBLIOGRAFÍA}

Alonso Santos, J.L. (2003): "Redes y procesos de innovación en las comarcas vinícolas de Castilla y León: el ejemplo de la D.O. Bierzo". Boletín de la Asociación de Geógrafos Españoles. № 36. Madrid. pp. 43-60

Andrés Sarasa, J.L. (2000):"Aportaciones básicas del turismo al desarrollo rural". Cuadernos de Turismo, no6, pp. 45-59.

Asero, V.; Patti, S. (2009): "From wine production to wine tourism experience. The case of Italy". American Asociation of Wine Economics. Consultada en www.wineeconomics.org el 15 de Mayo de 2010.

Aydeé Velásques, G. (2007): "Diseño de un modelo de sistema enoturístico para la gestión vitivinícola del Estado de Querétaro". Director: Dr. Ricardo Tejeira Padilla. Tesis para obtener el grado de Maestría en Ciencias en Alta Dirección de Empresas Turísticas.

Cánoves Valiente, G.; Herrera Jiménez, L.; Villarino Pérez, M. (2005): "Turismo rural en España: paisajes, nuevos usos y nuevas visiones". Cuadernos de Turismo, nํ15, pp 6376.

Cánoves, G.; Herrera, L.; Blanco, A. (2005): "Turismo rural en España: un análisis de la evolución en el contexto europeo". Cuadernos de Geografía, no77, pp. 41-58.

Del Canto Fresno, C.; García Álvarez, E. (2010): Puesta en valor del saber hacer vitivinícola y formación de capital social. La cooperación entre territorios: Ribera del 

del vino y sus consecuencias en la transformación del paisaje.

Duero (España) y Ain Temouchent (Argelia). En: Territorios Rurales. Pobreza, acción colectiva y multifuncionalidad. Claves e interrogantes sobre los sistemas agroalimentarios localizados. UNAM. México

Del Canto Fresno, C.; Ramírez García, S.; Freitas Caetano, S. (2011): Cuatro D.O.P vitivinícolas españolas: diferentes estrategias para un mismo espacio. En: Indications geographiques, dynamiques socio-economiques et patrimoine bio-culturel en Turquie et dans les pays mediterraneens. Montpellier: CIHEAM (Centre International de Hautes Etudes Agronomiques Méditerranéennes), (Séminaires Méditerranéens, Options Méditerranéennes).

Freitas Caetano, S.; Ramírez García, S.; Del Canto Fresno, C. (2011): Red de relaciones, cohesión social y dinámica territorial. El caso de la Denominación de Origen de Vinos de Uclés (Cuenca).

Goyet, P. (2010): "El turismo vitivinícola: el caso de Francia". Libro de actas del 2o Congreso Europeo de Turismo industrial. Toledo 2008. pp. 171-177

Hall, M., Sharples, L.; Cambourne, B.; Mancionis, N., (eds) (2000). "Wine tourism around the world: Development, management and markets", Elsevier Science, Oxford, UK.

López Guzmán-Guzmán, T.J., Sánchez Cañizares, S.M. (2008): “La creación de productos turísticos utilizando rutas enológicas". Pasos. Revista de Turismo y Patrimonio Cultural, Vol. 6, № 2, pp 159-171.

Matellanes Lazo, M. (2008): "Gestión del enoturismo en la D.O. Ribera del Duero de España". Directores: Dr. Salvador Hernández Navarro y Dra. Adriana Corrêa Guimarâes. Tesis doctoral para optar al grado de Doctora por la Universidad de Valladolid. Universidad de Valladolid, 2008.

Monnier, J.M. (2010): "La profesionalización del turismo vitivinícola: el ejemplo del Loira". Libro de actas del 2ㅇ Congreso Europeo de Turismo industrial. Toledo 2008. pp. 179-185

Parenteau, A. (2010): "Estado del turismo enológico en Europa". Libro de actas del 2은 Congreso Europeo de Turismo industrial. Toledo 2008. pp. 166-170

Pulido Fernández, J.I. y otros (2011): “El turismo rural en España. Orientaciones estratégicas para una tipología aún en desarrollo". Boletín de la Asociación de Geógrafos Españoles. № 56. Madrid. pp. 155-176

ISSN: 0212-8594 ISSN-e: 2340-2776 № DOI: http://dx.doi.org/10.12795/rea.2012.i29.04

REA 29 (2012):83-104

http://www.publius.us.es/estudios_andaluces 
Sánchez Hernández, J.L. (2003): “Capital exógeno y procesos de innovación en la industria vinícolas de la denominación de origen Toro". Boletín de la Asociación de Geógrafos Españoles. № 36. Madrid. pp. 61-80

Sáez, M. (2007): "El enoturismo en California". Notas Sectoriales del ICEX

Silva Pérez, R. (2002): "Estrategias de inserción de las áreas rurales en la economía mundial. Una aproximación desde Andalucía" Boletín de la Asociación de Geógrafos Españoles. № 33. Madrid. pp. 103-134

Simoes, O. (2008): "Enoturismo em Portugal: as Rotas de Vinho". Pasos. Revista de turismo y patrimonio cultural, Vol. 6, № 2, pp. 269-279.

Velasco González, M. (2009): "Gestión turística del patrimonio cultural: enfoques para un desarrollo sostenible del turismo cultural". Cuadernos de Turismo, no 23, pp. 237235.

Zarate Martín, M.A. (2010): La "visita de empresa" en España, una modalidad turística en expansión. Libro de actas del 20 Congreso Europeo de Turismo industrial. Toledo 2008. pp. 52-66.

\section{Recursos electrónicos:}

-Asociación Española de Ciudades del Vino de España: http://www.acevin.es/

-Consejo Regulador de la Denominación de Origen Yecla: www.yeclavino.com/

-Consejo Regulador de la Denominación de Origen Jumilla: www.vinosdejumilla.org/

-Consejo Regulador de la Denominación de Origen Bullas: www.vinosdebullas.es/

-Gran Selección de Vinos: http://www.granseleccion.com/shop/

-Red Europea de Ciudades del Vino: http://www.recevin.net/

-Rutas del Vino de España: http://www.wineroutesofspain.com/

-The Wine Institute: http://www.wineinstitute.org/

ISSN: 0212-8594 ISSN-e: 2340-2776 № DOI: http://dx.doi.org/10.12795/rea.2012.i29.04

REA 29 (2012):83-104

http://www.publius.us.es/estudios_andaluces 